\title{
COSTA RICA, EL REINO MOSQUITO Y EL “MULATO INTÉRPRETE” FRANCISCO CORELLA (1711-1724)
}

\author{
COSTA RICA, THE MOSQUITO KINGDOM, AND THE "MULATO \\ INTERPRETOR" FRANCISCO CORELLA (1711-1724)
}

Daniel Matthew Mendiola

Recibido: 25/05/2018 - Aceptado: 24/08/2018

\begin{abstract}
Resumen
Este artículo examina las relaciones entre la Costa Rica colonial y el Reino Mosquito, una confederación afroindígena que surgió en la costa caribeña de Centroamérica durante el siglo XVII. El objetivo de esta investigación es analizar el periodo de negociación durante la primera mitad del siglo XVIII, a través del estudio de los intermediarios que manejaban estas negociaciones en la práctica. Una figura central de esta narración es el "mulato intérprete" Francisco Corella, quien fue el principal negociador del año 1711 hasta al menos 1724. Además de contar la historia de estas negociaciones, este artículo demuestra las contribuciones importantes de poblaciones diversas, incluso personas afrodescendientes a la historia de Costa Rica.

Palabras clave: Reino Mosquito, Costa Rica, colonialismo, intermediario, negociación.

Abstract

This article examines the relationship between colonial Costa Rica and the Mosquito Kingdom: an afro-indigenous confederation that emerged on the Caribbean coast of Central America during the seventeenth century. The purpose of this investigation is to analyze a period of frequent negotiation during the early part of the eighteenth century by calling attention to the intermediaries who handled these interactions in practice. The central figure of this narrative is the "mulato interpreter" Francisco Corella who led these negotiations from 1711 until at least 1724. In addition to telling the story of these negotiations, this article demonstrates the important contributions of diverse populations, including people of African descent, to the history of Costa Rica.
\end{abstract}

Keywords: Mosquito Kingdom, Costa Rica, colonialism, intermediary, negotiation. 


\section{Introducción}

Este artículo examina las negociaciones entre Costa Rica y el Reino Mosquito durante el periodo que va de 1711 a 1729. Aunque estudios anteriores han supuesto que las relaciones entre estos dos pueblos siempre fueron violentas, los mosquitos y los costarricenses -en particular los habitantes de Matina que los mosquitos visitaban con frecuencia- fueron aliados durante la mayoría del siglo XVIII. La alianza ocurrió en dos etapas: la primera inició en el año 1711 y duró hasta más o menos el año 1740, y después de un corto periodo de hostilidades en el medio del siglo, una segunda época de paz empezó en el año 1763 y se extendió por el resto del siglo. Este ensayo describe la primera etapa de la alianza, la cual está bien documentada en una serie de informes y declaraciones actualmente guardados en el Archivo Nacional de Costa Rica (ANCR). De acuerdo con esas fuentes, este artículo narra los sucesos claves que dieron forma a la alianza.

Al relatar esta historia, este artículo se propone llamar la atención soobre el papel de los intermediarios: las personas que tenían contacto directo con los Mosquitos como representantes del pueblo español. Algunos servían como informantes después de haber vivido con los mosquitos como prisioneros. Otros desempeñaban el rol de diplomáticos o intérpretes durante visitas de los mosquitos a Matina. No obstante, lo que tenían en común es que, en estos papeles claves, los intermediarios ejercían mucha influencia de parte de los españoles, tanto en la formación de la política exterior hacia los Mosquitos como en la implementación de dicha política. En particular, este artículo se centra la vida de un intermediario llamado Francisco Corella, un "mulato" libre e intérprete del idioma mosquito quien ocupó una posición central en las negociaciones a lo largo de la primera etapa de paz. Aunque no ocupaba un puesto formal con el gobierno colonial, Corella hizo posible negociar con los mosquitos gracias a su conocimiento especializado e iniciativa personal.

El reconocimiento del papel de intermediarios como Francisco Corella es importante para enriquecer la comprensión de los procesos coloniales en las Américas. Aunque investigaciones históricas suelen mirar la historia "desde arriba" y atribuir los procesos del imperio a la dirección de funcionarios europeos, la verdad es que el proyecto colonial dependía mucho de la labor, conocimiento e iniciativa de diversas poblaciones. Dentro de esta dinámica, dos personas significativas fueron el Gobernador de Costa Rica Diego de la Haya Fernández, un español, y Francisco Corella, quien nació en Nicaragua y era considerado como "mulato" o "pardo" por su ascendencia africana. Mientras que el gobernador no habló directamente con los mosquitos ni una sola vez, Corella vivió varios años con los mosquitos como prisionero y cuando regresó a Matina después de ser liberado, se convirtió en embajador e intérprete principal para los españoles durante sus interacciones con los mosquitos. Su papel consistía en negociar directamente con líderes mosquitos durante sus visitas a Matina y, una vez, Corella encabezó una expedición diplomática que salió de Matina y llegó a la tierra 
de los Mosquitos. Por lo tanto, a pesar de la tendencia de contar esta historia como si el gobernador $\mathrm{u}$ otros funcionarios del gobierno lideraran las negociaciones, la verdad es que Diego de la Haya dependía completamente de intermediarios como Francisco Corella, quienes ejercían mucha influencia "desde abajo".

Aunque los intermediarios afrodescendientes tuvieron un papel vital en las relaciones entre Costa Rica y el Reino Mosquito, sus contribuciones no se encuentran fácilmente en los archivos de gobiernos superiores como el Archivo General de Centro América en Guatemala o el Archivo de las Indias en España. Sin duda, estas colecciones son muy importantes debido a su posición central en el mundo español, por lo que recopilaron documentos de muchos territorios. Sin embargo, estos archivos solían acumular resúmenes cortos e informes abreviados que favorecen la perspectiva de funcionarios como Diego de la Haya, ocultando a la vez el papel de los intermediarios como Francisco Corella. ${ }^{2}$ No obstante, las contribuciones de los intermediarios se observan con claridad en los informes originales y declaraciones completas que actualmente se encuentran en el ANCR. Por eso, las colecciones del ANCR ofrecen una oportunidad única para rastrear precisamente quién condujo las negociaciones en la práctica y también cómo se llevaron a cabo. Finalmente, recuperar la historia de intermediarios como Francisco Corella es preciso para entender cómo funcionaban el mundo colonial en realidad y, en particular, para llamar la atención hacia el papel de las personas afrodescendientes en la historia de Costa Rica.

\section{Antecedentes}

Para analizar las relaciones entre Costa Rica y el Reino Mosquito es necesario aclarar algunos detalles sobre el proyecto colonial de los españoles en Centroamérica. Durante los siglos XVII y XVIII, casi todo el territorio actual de Centroamérica pertenecía a la Audiencia de Guatemala, una jurisdicción constituida por las provincias de Guatemala, Honduras, Nicaragua, El Salvador y Costa Rica. El gobierno superior de la Audiencia se hallaba en Guatemala y era encabezado por el presidente nombrado por el rey de España. Bajo la supervisión del presidente, cada provincia tenía un gobernador a cargo de los funcionarios locales. En teoría, esta organización centralizaba el poder del rey sobre todo Centroamérica a través de un sistema jerárquico que facilitaba las órdenes desde arriba hacia abajo (Woodward; Elliott).

No obstante, en la práctica, la Audiencia se caracterizaba por la descentralización de poder, tanto por la autonomía del istmo frente a la Corona Española como por el aislamiento de muchas localidades fuera de la supervisión de Guatemala. Esta situación se basaba en realidades logísticas. Por ejemplo, el viaje entre España y Centroamérica duraba varios meses de forma tal que cada ronda de comunicación -enviar una orden y recibir una respuesta- podía tardar un año o más. Por eso, el presidente de Guatemala era suficientemente libre para formar sus propias políticas sobre 
asuntos cotidianos. Las pocas órdenes que llegaban de España no eran comprehensivas y, además, el presidente podía atrasar casi indefinidamente la implementación de órdenes reales, a través de trucos legales como pedir aclaración o alegar la imposibilidad de acatar las órdenes. También, la comunicación entre Guatemala y las demás provincias era muy lenta. Por eso, no deberíamos hablar sobre las políticas y las prácticas del mundo colonial de los españoles como si fuera una sola política compacta. En realidad, existía mucha variación, lo cual colocaba mucho poder en las manos de los actores locales (Woodward; Sellers-García).

Adicionalmente, el alcance de la autoridad española no comprendía los límites de la jurisdicción imaginada. Esta situación se basaba en los procesos de la conquista: mientras que las actividades de la conquista se enfocaban en la costa del Pacífico, los españoles carecían de gran influencia en el lado del Caribe, con la excepción de una pequeña cantidad de expediciones débiles que no lograron establecer asentamientos permanentes. Esta tendencia se mantuvo durante todo el siglo XVI de forma que, al inicio del siglo XVII, toda la costa caribeña, desde Trujillo en Honduras hasta Matina en Costa Rica, se hallaba libre de asentamientos e instituciones españoles (Pérez Brignoli; Ibarra).

Dentro de este contexto, la provincia más remota era Costa Rica, cuya capital, Cartago, estaba tan alejada de Guatemala que se requería un viaje de por lo menos tres semanas para enviar noticias desde una a la otra. Asimismo, la autoridad española dentro de la provincia de Costa Rica se limitaba a pocos sitios. La pequeña cantidad de habitantes de Costa Rica se concentraban en el Valle Central, lo cual dejaba muchas regiones sin asentamientos coloniales o hacía que aquellos que existían fuera del Valle Central se encontraran muy aislados. Para poner esta situación en perspectiva, el viaje entre Cartago y el Valle de Matina en la costa del Caribe tomaba de tres a cinco días para los mensajeros que llevaban noticias urgentes y de diez a doce días para arrieros llevando cargamento (Fonseca et al.).

En resumen, a pesar de sus pretensiones de haber conquistado todo el istmo, los españoles instalaron su autoridad solo sobre algunos territorios con una red tenue de asentamientos descentralizados. Por tanto, cuando varios grupos de indígenas y afrodescendientes en la Costa de los Mosquitos se unieron a finales del siglo XVII para fundar una confederación, este proceso ocurrió fuera del mundo español. En particular, la confederación se concentró alrededor del cabo Gracias a Dios en la costa caribeña de la actual Nicaragua. Se desconoce el año preciso de su formación, pero a mediados del siglo XVII, algunos grupos de mosquitos ya estaban involucrados en el mundo comercial del Caribe, proveyendo bastimentos a marineros europeos (Exquemelin). Adicionalmente, un llamado "rey" de los mosquitos visitó Jamaica en el año 1687 para formalizar sus relaciones diplomáticas y comerciales con los ingleses (The National Archives, Colonial Office 138/6, fol. 85). Por eso, algunas fuentes hacen referencia a un "Reino Mosquito", pero en la práctica el sistema de gobernación funcionó como una confederación (The National Archives, Colonial Office 132/1, fol. 52). Mientras el rey 
gobernó las inmediaciones del Cabo Gracias a Dios, otros cabos estaban a cargo sus propias regiones y se reunían con el rey frecuentemente para constituir un consejo central que consensuaba y ejecutaba acciones comunes.

Figura 1

MAPA DE LA EXPANSIÓN DE LOS MOSQUITOS APROXIMADAMENTE EN EL AÑO 1711

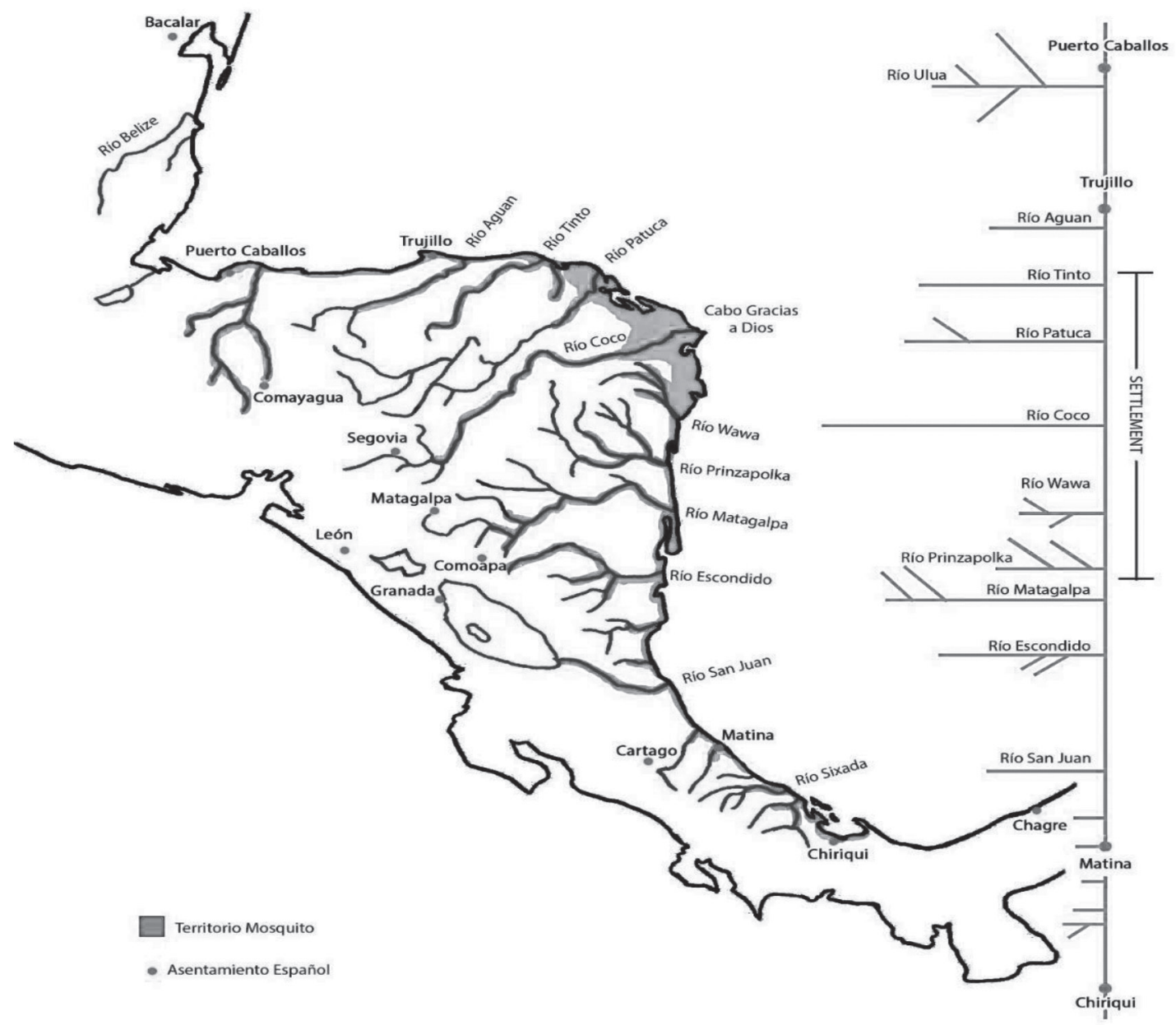

Fuente: Elaboración propia del autor, con ayuda de Sydnie Mares.

Al inicio del siglo XVIII, el Reino Mosquito se había convertido en una amenaza grave para el proyecto colonial de los españoles. Ya no atacaban a los españoles directamente, pero empezaron a atacar pueblos de indígenas y misiones en las zonas fronterizas de Honduras y Costa Rica. Con estas actividades, los mosquitos inspiraron 
nuevas migraciones de indígenas, dejando a los misioneros vulnerables y sin neófitos (Archivo General de Centro América, A1 (4), Legajo 50, Expediente 492, fol. 1). Adicionalmente, pusieron de relieve la vulnerabilidad de los asentamientos fronterizos. En centros coloniales como Granada y Santiago de Guatemala, los funcionarios superiores no demoraron en denunciar a los mosquitos, nombrándolos como enemigos sin excepción y pidiendo a la Corona apoyo para exterminarlos (Peralta). No obstante, la situación era distinta en Costa Rica, la provincia más cercana a la tierra de los Mosquitos y, por eso, la más vulnerable. Enfrentando una escasez de recursos militares, los funcionarios costarricenses entendían que no era posible exterminar a los mosquitos. Adicionalmente, junto a la amenaza de los mosquitos, los costarricenses vivían bajo la amenaza de otros indígenas también. Esta situación resulta evidente en el año 1710 cuando después de una sublevación de los indígenas de Talamanca se dio fin al proyecto de cristianización (Fernández). Por eso, los costarricenses no estaban completamente de acuerdo con la política de Guatemala y, en la práctica, estaban abiertos a otras opciones.

Esta era la situación en al año 1711, cuando una flota de mosquitos llegó a Matina para proponer un acuerdo de paz. Considerando la proposición como una oportunidad de reducir las amenazas enfrentadas por la vulnerable provincia, los funcionarios de Cartago hicieron caso a la propuesta a pesar de la opinión negativa hacia los mosquitos por parte del gobierno superior. En Cartago, esta política continuó siendo un tema de debate, con algunos funcionarios a favor de una alianza con los mosquitos y otros en contra. Sin embargo, en Matina, el asunto fue decidido en la práctica: a partir del año 1711, las flotas de mosquitos fueron recibidos pacíficamente como aliados. Por lo tanto, mientras que gobiernos superiores continuaron debatiendo las implicaciones de la expansión de los mosquitos de forma teórica, un cadre de intermediarios llevó a la práctica una alianza entre Costa Rica y el Reino Mosquito.

\section{Francisco Corella y el inicio de las negociaciones en Matina: 1711-1718}

Francisco Corella nació en el pueblo de Managua en la provincia de Nicaragua a finales del siglo XVII. Según documentos oficiales, Corella era "pardo" o "mulato" libre; esto significa que era afrodescendiente, pero no era un esclavo. Cuando aún era joven, se mudó a Matina y consiguió trabajo en una hacienda de cacao (ANCR, Guatemala (GA)-144, fol. 32). No se sabe precisamente cuánto tiempo trabajó en Matina, pero alrededor del año 1708, Corella fue secuestrado por un grupo de mosquitos durante una breve incursión en el valle. Corella fue un esclavo en la tierra de los mosquitos por cinco años, pero impresionó a sus captores con su inteligencia y su habilidad para los idiomas. Por este motivo, Corella ganó el respeto del rey de los Mosquitos, Bernabé, quien sirvió como intérprete (ANCR, Cartago (CO)-192, fol. 103). 
En el año 1711, el Rey Bernabé emprendió una nueva estrategia diplomática, la cual incluyó proponer paz a varias comunidades españolas. En particular, envió recados a las provincias de Honduras, Nicaragua y Costa Rica. La proposición no tuvo mucho efecto en Nicaragua, pero hay evidencia de que el Puerto de Trujillo en la costa de Honduras sí aceptó el tratado por lo menos informalmente (ANCR, GA-144, fol. 3-5). No obstante, la estrategia fue muy efectiva en Costa Rica, donde inició una larga etapa de alianzas con los habitantes de Matina. Las negociaciones comenzaron en junio cuando una flota de mosquitos llegó a Matina y liberó varios prisioneros incluyendo a un "negro" llamado Reimundo y a una mujer indígena de la región de Chontales con recado verbal de parte del Rey Bernabé (ANCR, GA-144, fol. 7). Las descripciones de este acontecimiento no confirman claramente si Corella asistió, pero sí tuvo un rol en las negociaciones, pues conoció a los otros prisioneros cuando todavía estaban en la tierra de los mosquitos y, siendo intérprete, les explicó los términos de la proposición de Bernabé. Con esta ayuda brindada por Corella, el plan de Bernabé funcionó y los prisioneros liberados reportaron que la propuesta de Bernabé era sincera (ANCR, GA-144, 9-13).

En Cartago, el gobernador escribió un resumen de los testimonios y lo entregó al presidente en Guatemala, con el fin de pedir la aprobación del tratado. Considerando la vulnerabilidad de Matina, los costarricenses vieron el ofrecimiento como una manera de apaciguar a los mosquitos y reducir el peligro enfrentando por la provincia. Sin embargo, la decisión oficial, escrita por el fiscal de Guatemala, ordenó al Gobernador de Costa Rica que rechazara la proposición y tratara a los mosquitos como enemigos de la Corona. Para el fiscal, el problema se hallaba en asuntos de comercio. Ya que otros informes habían reportado que los mosquitos tenían relaciones comerciales con los ingleses, cualquier intercambio con los mosquitos sería considerado contrabando (ANCR, GA-144, fol. 19). Entonces, sin garantías de la suspensión de este comercio, el tratado entre los mosquitos y Costa Rica no sería válido.

No obstante, los costarricenses no hicieron caso a las órdenes y cuando otra delegación de mosquitos llegó a Matina en el año 1713, fueron recibidos con amistad. Durante esta segunda ronda de negociaciones, Francisco Corella fue liberado con el fin de actuar como delegado del Rey Bernabé (ANCR, CO-192, fol. 1, 6-7). Corella llegó con una flota de mosquitos encabezada por el Gobernador Aníbal y el General Peter, dos líderes vinculados con Bernabé al ostentar puestos en el consejo central de la confederación mosquita. La flota llegó en junio de 1713 y, después de un cordial encuentro -facilitado por el intermediario Francisco Corella en el papel de intérprete-, la flota continuó su viaje hacia el sur mientras Corella viajó a Cartago para sostener una entrevista con el Gobernador de Costa Rica. A pesar de haber sido secuestrado, Corella habló bien de los mosquitos y le aseguró al gobernador que Bernabé deseaba una alianza verdadera. Por eso, su testimonio se convirtió en la base de una apelación para que el gobierno superior de Guatemala reconociera el tratado. Por ejemplo, Corella abordó 
el tema de comercio, el cual había sido la razón para rechazar la proposición dos años antes. Según la declaración, los mosquitos no tenían un acuerdo permanente con los ingleses así que eran libres para comerciar exclusivamente con los españoles. También, Corella mencionó el deseo de los mosquitos de convertirse al catolicismo, otro requisito común para legalizar acuerdos con grupos indígenas (ANCR, CO-192, fol. 1-3). La flota regresó en julio y fue bien recibida nuevamente, pero los funcionarios costarricenses le informaron que no podían finalizar el tratado porque todavía estaban esperando una repuesta de Guatemala (ANCR, CO-192, fol. 16; ANCR, GA-144, fol. 34-35).

El gobierno superior de Guatemala no envió respuesta hasta noviembre. A pesar de la declaración de Corella y la solicitud del gobernador a favor de aceptar a los mosquitos como aliados, los funcionarios de Guatemala repitieron los órdenes de tratar a los mosquitos como enemigos. Esta decisión igualmente se basó en asuntos de comercio. Desde la perspectiva de Guatemala, el testimonio de Corella no era suficiente garantía de que los mosquitos iban a detener su comercio con los ingleses. Por lo tanto, la política oficial de Guatemala se mantenía en contra de los mosquitos (ANCR, CO-192, fol. 20). No obstante, aunque el Gobernador de Costa Rica no pudo aceptar el tratado con los mosquitos oficialmente, el gobernador tampoco aplicó medidas para interrumpir la paz, dejando el asunto en las manos de los habitantes de Matina. De esta manera, Matina y el Reino Mosquito establecieron un tratado informal en la práctica. Mientras tanto, Francisco volvió a vivir en Matina, sirviendo como intérprete y negociante principal por parte de los costarricenses.

\section{Diego de la Haya Fernández e interrupciones de la paz: 1719-1724}

La próxima visita documentada de una delegación de mosquitos a Matina sucedió en el año 1719, pero es posible que existieran otras visitas en años anteriores que no fueron reportadas. Ya que Guatemala consideraba ilegal cualquier trato con los mosquitos, habría sido más conveniente esconder las visitas para prevenir acusaciones de contrabando. En cualquier caso, es cierto que los mosquitos y los costarricenses mantuvieron la paz durante estos años, sin que ninguna de las partes participara de actos hostiles contra la otra. Adicionalmente, cuando una flota pequeña de mosquitos llegó a Matina en enero de 1719 pidiendo bastimentos -un acontecimiento documentado por el teniente de Matina- los visitantes fueron recibidos como si fuera un suceso normal. Ninguno de los líderes principales de la confederación estuvo presente, pero los marineros de la flota aseguraron a los habitantes de Matina que muy pronto el Rey Bernabé visitaría la zona para reiterar sus propuestas previas (ANCR, CO-219, fol. 1). Este informe era preciso y Bernabé llegó a Matina en abril, tres meses después, a la cabeza de una flota masiva. La flota había venido de Bocas del Toro en el sur e iba de regreso a la tierra de los mosquitos al norte, llevando ciento cincuenta soldados y ochenta esclavos indígenas recién capturados. Durante la visita, Francisco Corella sirvió como intérprete 
y diplomático, negoció directamente con Bernabé y relató los términos del acuerdo al alférez de Matina. Al fin, Bernabé prometió a Corella que regresaría con los demás líderes de la confederación para finalizar el pacto (ANCR, CO-223, fol. 2-5).

Desde la perspectiva de Matina, la visita fue un éxito, aseguró que la paz de los años anteriores continuaría. Sin embargo, la situación era más complicada en Cartago, ya que recientemente había llegado un nuevo gobernador: el español Diego de la Haya Fernández. Aunque los gobernadores anteriores habían disfrutado la falta de supervisión de gobiernos superiores para aplicar de manera flexibles las leyes inconvenientes, el gobernador de la Haya llegó con la intención de aplicar la ley rigorosamente incluso en asuntos de contrabando. Por tanto, no estaba satisfecho con un acuerdo informal y cuando se enteró de que el Rey Bernabé visitaría Matina, el gobernador fijó un estándar muy alto para los términos del tratado. Por lo tanto, aunque la visita de Bernabé en abril de 1719 reforzó la paz de forma práctica en Matina, este era un asunto pendiente desde la perspectiva del gobernador Diego de la Haya (ANCR, CO-219, fol. 3).

Este factor afectó las negociaciones de cierto modo, pero es preciso reiterar que para el gobernador las negociaciones eran abstractas: nunca habló con los mosquitos durante sus visitas y siempre dependió de otros para recibir información sobre los sucesos. Él participó en las negociaciones indirectamente, a través de la exposición de su política, pero su influencia era limitada y no siempre podía imponer sus demandas completamente "desde arriba". Esto resultó evidente el año siguiente, cuando llegó a Matina otra flota de mosquitos en el mes de abril. Ya que el Rey Bernabé había dado su palabra de que iba a regresar con los demás líderes de la confederación, Diego de la Haya tenía la esperanza de poner fin a la cuestión del tratado a través de la presentación clara de todos los requisitos de la ley. Sin embargo, Bernabé no volvió y, en su lugar, el gobernador de los mosquitos, llamado Aníbal, vino a cargo de la flota. Por eso, aunque los visitantes reiteraron su intención de mantener la paz, no pudieron formalizar el tratado con el alto estándar fijado por Diego de la Haya. Entonces, el asunto de la paz estaba aún irresuelto pendiente y creció la desconfianza del gobernador con respecto a la sinceridad de los mosquitos y los habitantes de Matina. Por eso, el gobernador intentó reiterar desde lejos que el tratado era ilegal y dio órdenes de investigar que nadie hubiera comerciado con los mosquitos durante la visita (ANCR, CO-272, fol. 9).

En contraste, mientras el gobernador Diego de la Haya quedó preocupado por la política teórica, Francisco Corella continuó su papel de intermediario en la práctica, reiterando a los mosquitos las buenas intenciones de los costarricenses y recopilando inteligencia sobre las actividades de los mosquitos. De esta manera, el gobernador dependía de los intermediarios y después de la visita de Aníbal, Corella viajó a Esparza para explicar lo que había pasado. Junto a Corella, viajó otro intérprete recién liberado de los mosquitos, un "negro esclavo" llamado Miguel que había sido secuestrado en Matina alrededor del año 1703. Los dos informantes testificaron que la confederación mosquita todavía quería paz con los costarricenses y que la 
ausencia de Bernabé no representaba un problema para las negociaciones. De hecho, reportaron que Bernabé y Aníbal habían rechazado planes de comerciantes ingleses para saquear Matina, debido a que el valle costero estaba bajo la protección del Reino Mosquito (ANCR, CO-272, fol. 11-13).

Las acciones de los mosquitos, después de esta visita, demostraron que Francisco Corella y los habitantes de Matina los habían convencido de las intenciones de los costarricenses para continuar la alianza. Por ejemplo, la flota de Aníbal regresó a Matina para otra visita dos semanas más tarde, en mayo de 1720, después de haber apresado dos barcos de corsarios franceses (ANCR, CO-272, fol. 18). También, otra flota de mosquitos llegó a Matina en abril del año 1721 para recoger bastimentos y esperar que se reuniera con otra flota encabezada por Aníbal. En esta ocasión Francisco Corella también fue el negociante principal, habló con el capitán de la flota sobre su itinerario y arregló los bastimentos (ANCR, CO-282, fol. 18).

Sin embargo, a pesar de los esfuerzos de Corella para mantener la alianza informal, la desconfianza del gobernador De la Haya creció aún más. Sin el establecimiento de un tratado formal con Bernabé y los demás líderes, De la Haya sospechaba que los mosquitos iban a traicionar a Costa Rica. Este temor aumentó en enero de 1721, cuando el gobernador recibió un informe de Nicaragua en el cual se alegaba que los mosquitos estaban construyendo una flota de cincuenta canoas de guerra para invadir Matina. Por eso, Diego de la Haya mandó más refuerzos a Matina con órdenes de prepararse para un ataque sorpresivo (ANCR, CO-282, fol. 3, 11).

Esta intervención del gobernador De la Haya casi causó el estallido de hostilidades en octubre de 1721. El encuentro aconteció cuando Aníbal llegó a Matina con otra flota grande para continuar su campaña contra los corsarios franceses. Después de más de diez años de utilizar el puerto de Matina como aliado, Aníbal no anticipó ningún problema y se acercó al puerto para recoger bastimentos y compartir inteligencia. No obstante, los nuevos soldados de la vigía, enterados de los informes de Nicaragua en los cuales se alegaba la perfidia de los mosquitos, anticiparon una emboscada y abrieron fuego contra la delegación (ANCR, CO-286, fol. 1-4). Sorprendido y furioso, Aníbal retrocedió a la flota para preparar una represalia. Afortunadamente para los habitantes de Matina, Francisco Corella llegó a tiempo para frenar la escalada de tensiones. Junto con el teniente general de Matina y otro "mulato" intérprete llamado Diego de Bonilla, Corella salió en canoa bajo señas de paz para hablar con Aníbal más allá de la costa. Los negociantes lograron reducir la tensión y Aníbal no atacó, pero demandó a los costarricenses más garantías de que sería reconocido su derecho de visitar Matina en el futuro (ANCR, CO-286, fol. 37-38).

Después de la junta entre Francisco Corella, Diego de Bonilla y Aníbal, las vigías permitieron que unas canoas mosquitas se acercaran al puerto para recoger bastimentos y la flota continuó hacia el sur con un compromiso de regresar pronto. Entonces, Corella se quedó en Matina y Bonilla fue a Cartago con otros funcionarios 
de Matina para informar al gobernador de lo sucedido (ANCR, CO-286, fol. 7). Durante las declaraciones, los testigos dieron argumentos a favor de Aníbal, afirmaron que los mosquitos no habían causado ningún daño en Matina desde el inicio de la paz en 1711, al contrario, que las flotas de los mosquitos habían protegido el valle contra piratas (ANCR, CO-286, fol. 27-28). Esto eventualmente convenció al gobernador, quien firmó una patente para nombrar oficialmente a Aníbal capitán de guarda costas del Valle de Matina (ANCR, CR-286, fol. 46 ,57-58). El día 1 de noviembre, Diego de la Haya entregó Matina con instrucciones de que Francisco Corella la presentara a Aníbal cuando este arribara. Esto ocurrió muy pronto, el día 10 de noviembre. Esta vez la flota fue recibida pacíficamente y Francisco Corella presentó la patente a Aníbal para consolidar la alianza. Al final, Aníbal prometió regresar dentro de unos meses con Bernabé y Peter para finalizar el tratado con representantes de toda la confederación (ANCR CO-290, fol. 4-5).

A pesar de esta resolución provisional, la desconfianza de Diego de la Haya empezó a crecer nuevamente el año siguiente, cuando Aníbal no cumplió con su compromiso de regresar dentro del plazo previsto. Entonces, en marzo de 1722, el gobernador repitió su orden del año anterior para enviar nuevas tropas a Matina en anticipación de un ataque (ANCR, CO-290, fol. 4-5). Sin embargo, los informantes con información de primera mano continuaban alegando las buenas intenciones de los mosquitos. Por ejemplo, durante el mismo mes de marzo, un prisionero fugitivo de los mosquitos -un "negro congo" llamado Antonio- testificó ante el gobernador que Bernabé y los demás líderes todavía deseaban regresar para finalizar el tratado, pero estaban atrasados por la necesidad de construir nuevas canoas para la flota (ANCR, CO-290, 1-3). Asimismo, en lugar de interpretar el retraso de Aníbal como pérfida, Francisco Corella se mantuvo a favor de la alianza y para comprobar las buenas intenciones de los mosquitos, encabezó una expedición diplomática que salió de Matina el día 9 de abril y llegó a la casa de Aníbal el día 19 (ANCR, CO-290, fol. 7, 32-33). Durante la visita, Corella asistió a una junta con Bernabé, Aníbal y Peter donde los tres líderes firmaron una carta, confirmando que todavía querían paz y que no habían regresado a tiempo a Matina por causa de problemas logísticos (ANCR, CO-290, fol. 35). Con esta carta, la expedición de Corella regresó a Matina el día 29 de mayo, con una escolta encabezada por el cuñado de Aníbal (ANCR, CO-290, fol. 30-31).

En Matina, el cuñado de Aníbal fue recibido como héroe y los habitantes del valle interpretaron la carta como una resolución final. No obstante, cuando Corella fue a Cartago para llevar la carta y testificar sobre la expedición, el gobernador reaccionó de manera diferente. El problema fue que además de confirmar sus intenciones de mantener la paz con Costa Rica, la carta de los líderes mosquitos también admitía que la confederación no iba a suspender sus vínculos comerciales con los ingleses de Jamaica. Por su parte, Corella intentó convencer al gobernador de que sería deseable apaciguar a la poderosa confederación y aceptar sus términos a pesar de su trato con 
los ingleses (ANCR CO-290, fol. 41-42). No obstante, Diego de la Haya decidió finalmente rechazar la proposición y envió órdenes a Matina para que suspendieran todo contacto con los mosquitos. De igual forma, con el fin de a asegurar que sus órdenes tuvieran efecto, envió refuerzos bajo el mando de un teniente leal, quien apresó y reemplazó al anterior teniente de Matina (ANCR, CO-290, fol. 53, 76).

Estas acciones del gobernador en Cartago cesaron oficialmente las negociaciones que habían existido por más de diez años. Sin embargo, los intermediarios de Matina intentaron mantener la paz a pesar de esta grave provocación por parte de los costarricenses. Desafortunadamente para Matina fue imposible frenar completamente la escalada de tensiones; sin embargo, al demostrar la influencia de estos intermediarios, lograron prevenir el estallido de hostilidades durante más de un año. Estas acciones fueron bien documentadas durante una visita que sucedió en enero de 1723, la primera visita después de la aplicación de la nueva política. Cuando la flota se acercó al puerto con la expectativa de recibir bastimentos, como en otros años, el capitán se enojó al ser rechazado y demandó hablar directamente con el intérprete Diego de Bonilla. Contra las órdenes de suspender todo contacto, Bonilla se reunió con el capitán y lo convenció de continuar su viaje sin atacar (ANCR, CO-295, fol. 92-94). Después de este encuentro, la delicada paz duró un año más.

Sin embargo, la nueva política hacia los mosquitos eventualmente provocó una represalia. Lo anterior ocurrió en abril de 1724, cuando un ejército de cientos soldados mosquitos encabezados por Aníbal lanzó una invasión sorpresiva, con lo cual detuvieron la milicia casi sin resistencia y ocuparon el valle a lo largo de tres días. Durante este plazo, las tropas mosquitas confiscaron una gran porción de la cosecha de cacao; luego, se la llevaron en dos barcos junto con sus canoas de guerra. También tomaron docenas de prisioneros (ANCR, CO-303, fol. 17-19). No obstante, a pesar de causar tanto daño, Aníbal dejó un recado el cual explicaba que él todavía quería ser aliado de Matina y que la invasión fue simplemente un castigo por la traición de haber suspendido relaciones diplomáticas. De hecho, Aníbal ofreció liberar a los prisioneros y demandó que Francisco Corella y Diego de Bonilla vinieran a la tierra de los mosquitos de nuevo para renegociar el tratado (ANCR, CO-303, fol. 21). Con esta acción, la flota se marchó; los costarricenses se vieron en la obligación de pensar cómo prevenir otro desastre en el futuro.

\section{Conclusiones}

Después de la invasión, Diego de la Haya se mantuvo obstinado en su posición, se negó a permitir que Francisco Corella emprendiera otra expedición diplomática o a permitir el pago del rescate (ANCR, CO-310, fol. 1-4). No obstante, el castigo de Aníbal tuvo efecto en Matina y a partir de entonces sus habitantes volvieron a recibir flotas de los mosquitos y a proveer bastimentos. Por ejemplo, cuando Aníbal regresó a 
Matina en enero de 1725, el puerto lo recibió pacíficamente y lo agasajó con cantidades de plátano y cacao (ANCR, CO-310, fol. 1-4). Igualmente, cuando Bernabé visitó Matina dos meses después para ofrecer el rescate de los prisioneros, su flota fue recibida pacíficamente (ANCR, CO-313, fol. 43-47). De esta manera, la alianza entre los mosquitos y Matina se estableció de nuevo en la práctica y duró hasta el fin de la década.

Entre los años 1726-1730, delegaciones de mosquitos llegaron a Matina por los menos diez veces más y, aunque los documentos del ANCR describen cada una de estas visitas, el nombre Francisco Corella desapareció de las fuentes. Es probable que él continuara sus actividades de intermediario sin ser mencionado en los informes, pero también es posible que el dejara de participar del todo. No obstante, es cierto que los precedentes establecidos bajo su liderazgo en años anteriores continuaron dando forma a las negociaciones de paz en la práctica, lo cual hizo posible que Matina mantuviera relaciones pacíficas con el Reino Mosquito, a pesar de la presión de gobiernos superiores para suspender todo contacto.

En última instancia, la historia de Francisco Corella y los demás intermediarios pone de relieve la forma en que el proyecto colonial de los españoles dependía de diversos actores. La negociación entre Costa Rica y el Reino Mosquito es solo un ejemplo, pero cualquier iniciativa requería actores, tanto por su conocimiento especializado como para llevar las acciones a la práctica. Por lo tanto, es importante que los historiadores busquen estos actores en fuentes escritas, primero para entender cómo el colonialismo funcionaba en realidad y, en segunda instancia, para para llamar la atención hacia las contribuciones de actores afrodescendientes, indígenas y de cualquier otra pertenencia de forma paralela a la de los europeos.

\section{Notas}

1 Para ejemplos de estudios que favorecen la perspectiva de Diego de la Haya para interpretar las relaciones con los mosquitos (Salvatierra 422-423; Floyd 68; Chacón de Umaña 83-95; Thornton 18).

Para un análisis más comprensivo de la historiografía y el efecto de ciertas fuentes primarias (Mendiola, 2018).

\section{Bibliografía}

Chacón de Umaña, Luz Alba. Don Diego de la Haya Fernández. Editorial Costa Rica, 1968.

Elliott, J. H. Imperial Spain, 1469-1716. St. Martin's Press, 1964.

Exquemelin, A. O. The Buccaneers of America: A True Account of the Most Remarkable Assaults Committed of Late Years upon the Coasts of the West Indies by the Buccaneers of Jamaica and Tortuga. Cambridge University Press, 2010.

Fernández, León. Colección de Documentos para la Historia de Costa Rica. Vol. IX. Imprenta Nacional, 1881.

Floyd, Troy S. The Anglo-Spanish Struggle for Mosquitia. University of New Mexico Press, 1967. 
Fonseca, Elizabeth C., et al. Costa Rica en el siglo XVIII. Editorial de la Universidad de Costa Rica, 2001.

Ibarra, Eugenia. Del arco y la flecha a las armas de fuego: Los indios mosquitos y la historia centroamericana 1633-1786. EUCR, 2011.

Mendiola, Daniel. "The Rise of the Mosquito Kingdom in Central America's Caribbean Borderlands: Sources, Questions, and Enduring Myths". History Compass, 16.1 (2018). doi:10.1111/hic3.12437

Peralta, Manuel. Ed. Costa Rica y costa de Mosquitos, documentos para la historia de la jurisdicción territorial de Costa Rica y Colombia. Lahure, 1898.

Pérez Brignoli, Héctor. A Brief History of Central America. University of California Press, 1989.

Salvatierra, Sofonías. Contribución a la historia de Centroamérica. Tip. Progreso, 1939.

Sellers-García, Sylvia. Distance and Documents at the Spanish Empire's Periphery. Stanford University Press, 2014.

Thornton, John K. "The Zambos and the Transformation of the Miskitu Kingdom, 1636-1740". Hispanic American Historical Review, 97.1 (2017): 1-28. doi: 10.1215/00182168-3727364.

Woodward, Ralph Lee. Central America: A Nation Divided. Second edition. Oxford University Press, 1985.

\section{Fuentes primarias manuscritas}

Archivo General de Centro América. A1 (4), Legajo 50, Expediente 492, fol. 1. “Carta de Lázaro de Castro". Villa de San Cruz de Yoro, 1705.

Archivo Nacional de Costa Rica (ANCR). Cartago (CO)-192, fol. 1-3. “Declaración de Francisco Corella prisionero". Cartago, 3 de julio de 1713.

ANCR. CO-192, fol. 1. "Carta de Joseph Antonio Lacayo de Briones". Cartago, 3 de julio de 1713.

ANCR. CO-192, fol. 6-7. "Autos". Cartago, 8 de julio de 1713.

ANCR. CO-192, fol. 16. "Carta de Francisco de Ibarra". Matina, 31 de julio de 1713.

ANCR. CO-192, fol. 20. "Carta del fiscal”. Santiago de Guatemala, 6 de noviembre de 1713.

ANCR. CO-219, fol. 1. "Carta de Francisco Espinoza a Diego de la Haya Fernández," Matina, 3 de enero de 1713.

ANCR. CO-219, fol. 3. "Auto de Diego de la Haya Fernández". Cartago, 14 de enero de 1719.

ANCR. CO-223, fol. 2. “Carta de Felipe de Mesa”. Matina, 11 de abril de 1719.

ANCR. CO-223, fol. 4-5. "Declaración del alférez Miguel del Castillo de edad de 24 años", Cartago, 24 de abril de 1719.

ANCR. CO-272, fol. 9. “Orden de Diego de la Haya Fernández". Esparza, 28 de abril de 1720.

ANCR. CO-272, fol. 11-12. “Declaración de Miguel del Camino, negro esclavo". Esparza, 7 de mayo de 1720.

ANCR. CO-272, fol. 12-13. “Declaración de Francisco Corella mulato español e ynterprete de la lengua Mosquito". Esparza, 7 de mayo de 1720.

ANCR. CO-272, fol. 1. "Carta de Antonio de Arlegui". Matina, 6 de mayo de 1720.

ANCR. CO-282, fol. 3. "Auto de Diego de la Haya Fernández". Cartago, 20 de enero de 1721 y

ANCR. CO-282, fol. 11. "Junta de Guerra". Cartago, 21 de enero de 1721.

ANCR. CO-282, fol. 76. "Carta de Alvaro de Quevara y Joseph de Bonilla a Diego de la Haya Fernández". Matina, 3 de abril de 1721.

ANCR. CO-282, fol. 78. "Junta de guerra". Cartago, 18 de abril de 1721. 
ANCR. CO-286, fol. 1-2: carta de Bernardo Pacheco, Pedro Ximenez, and Joseph de los Rios a Diego de la Haya Fernández, Matina, 10 de octubre de 1721.

ANCR. CO-286, fol. 3-4. "Declaración de Don Manuel de Rojas". Matina, 10 de octubre de 1721.

ANCR. CO-286, fol. 7. "Auto de Diego de la Haya Fernández". Cartago, 16 de abril de 1721.

ANCR. CO-286, fol. 27-28. "Parecer". Cartago, 17 de abril de 1721.

ANCR. CO-286, fol. 37-38. "Auto de Sargento Manuel Arlegui". Cartago, 18 de octubre de 1721.

ANCR. CO-286, fol. 46. "Notificación al Sargento Mayor Manuel Arlegui". Cartago, 28 de octubre de 1721 .

ANCR. CO-286, fol. 57-58. "Testimonio de la patente para gov. Anibel". Cartago, 1 de noviembre de 1721.

ANCR. CO-286, fol. 63-65. "Carta de Bernardo Pacheco a Diego de la Haya Fernández". Matina, 18 de noviembre de 1721.

ANCR. CO-290, fol. 1-3. "Declaración del negro congo Antonio de edad al parecer 32 años". Cartago, 8 de marzo de 1722.

ANCR. CO-290, fol. 4-5. "Orden de Diego de la Haya Fernández". Cartago, 8 de marzo de 1722.

ANCR, CO-290, fol. 7. “Carta de Francisco Ibarra a Diego de la Haya Fernández”. Matina, 9 de abril de 1722.

ANCR, CO-290, fol. 32-33. "Carta de Joseph de los Rios y Francisco Corella a Francisco Ybarra". Mosquitia, 25 de abril de 1722.

ANCR. CO-290, fol. 30-31. "Carta de Bernardo Pacheco a Diego de la Haya Fernández". Matina, 29 de mayo de 1722.

ANCR. CO-290, fol. 35. “Carta del Rey Don Bernabé, el Gobernador Aníbal, y el General Don Pedro a Diego de la Haya Fernández". Mosquitia, 11 de mayo de 1722.

ANCR. CO-290, fol. 41-42. "Declaración de Francisco Corella pardo libre”. Cartago, 8 de junio de 1722.

ANCR. CO-290, fol. 53. “Orden de Diego de la Haya Fernández". Cartago, 9 de junio de 1722.

ANCR. CO-290, fol. 76. "Carta de Geronimo Manuel Ramos a Diego de la Haya Fernández". Matina, 25 de junio de 1722.

ANCR. CO-295, fol. 92. "Carta de Domingo de Quesada a Diego de la Haya Fernández". Matina, 12 de enero de 1723.

ANCR. CO-295, fol. 94. "Carta de Domingo de quesada a Diego de la Haya Fernández". Matina, 16 de enero de 1723.

ANCR. CO-303, fol. 17. “Carta de Don de Quesada a Diego de la Haya Fernández”. Matina, 20 de abril de 1724 .

ANCR. CO-303, fol. 19. "Carta de Pedro de Molina a Don de Quesada". Matina, 19 de abril de 1724.

ANCR. CO-303, fol. 21. “Carta de Aníbal al Captain del Valle Domingo de Quezada”. Matina, 19 de abril de 1724.

ANCR. CO-310, fol. 1-2. “Declaración de Joseph Antonio”. Cartago, 17 de enero de 1725.

ANCR. CO-310, fol. 3-4. “Declaración de Julián Solano". Cartago, 17 de enero de 1725.

ANCR. CO-313, fol. 41. “Orden de Diego de la Haya Fernández". Cartago, 5 de marzo de 1725.

ANCR. CO-313, fol. 43-45. "Carta de Garido a Diego de la Haya Fernández". Matina, 30 de marzo de 1725. 
ANCR. CO-313, fol. 46-47. "Carta de Alvarado de Jirales a Diego de la Haya Fernández". Matina, 30 de marzo de 1725.

ANCR. Guatemala (GA)-144, fol. 3-5. "Carta Antonio Granda de Balbín”. Cartago, 4 de julio de 1711.

ANCR. GA-144, fol. 7. "Carta de Joseph Carasola y Cordova”. Cartago, 1 de julio de 1711.

ANCR. GA-44, fol. 9-13. "Declaración de Francisco Jimenez". Cartago, 26 de junio de 1711.

ANCR. GA-144, fol. 19. "Auto de Fiscal Joseph Guthierrez". Santiago de Guatemala, 20 de agosto de 1711.

ANCR. GA-144, fol. 32. "Carta de Joseph Antonio Lacayo de Briones". Cartago, 12 de agosto de 1713.

ANCR. GA-144, fol. 34-35. "Joseph Antonio Lacayo de Briones”. Cartago, 17 de agosto de 1713.

The National Archives (TNA). Colonial Office 138/6, fol. 85. “Letter from Duke of Albermarle to the Committee". Jamaica, 11 de febrero de 1688.

TNA. Colonial Office 123/1, fol. 52. "Declaration of King Edward of the Mosquito Indians". Dakura, 16 de marzo de 1740.

Daniel Matthew Mendiola. Estadounidense. Doctor en Historia de América Latina, con énfasis en historia colonial, por la Universidad de Houston. Su especialización es la costa caribeña de Centroamérica y la Mosquitia. Es becario por el programa Fulbright para realizar una estancia de investigación en el Archivo Nacional de Costa Rica.

Contacto: danielmendiola23@gmail.com

ORCID: 0000-0002-4175-6280 